\author{
Christopher Uhlig \\ Thomas Felbinger \\ Axel R. Heller
}

\section{Comments on Kagan et al.: Preemptive enteral nutrition enriched with eicosapentaenoic acid, gamma-linolenic acid and antioxidants in severe multiple trauma: a prospective, randomized, double-blind study}

Accepted: 14 June 2015

Published online: 25 June 2015

(C) Springer-Verlag Berlin Heidelberg and ESICM 2015

Dear Editor,

We read with great interest the manuscript by Kagan and colleagues regarding early enteral nutrition enriched with omega-3 polyunsaturated fatty acids in intensive care unit patients suffering from severe multiple trauma [1].

In severe trauma, systemic inflammatory response may induce multiorgan failure. Omega-3 polyunsaturated fatty acids ( $\omega-3$ PUFAs) have shown to resolve the effects in ARDS [2] and sepsis [3]. The key concept of $\omega-3$ PUFA use is limitation of the overwhelming inflammatory reaction at the earliest possible point in time to cover the peak of the inflammatory response in the first 3-4 days after onset of trauma. The integration of $\omega$-3 PUFAs into the cellular membrane is the precondition for their metabolism which results in the desired anti-hyperinflammatory effects. Thus, administration $\omega-3$ PUFAs must commence at the earliest possible point in time.

As demonstrated earlier [4], the intestinal resorption of $\omega$-3 PUFAs is not sufficient in all patients during systemic inflammation. The enteral application form chosen in the study protocol led to too slow a buildup of $\omega-3$ PUFA pool with respect to the chosen enteral dosage. Not till the fourth day was a measurable increase of $\omega$-3 PUFA detected in the cellular membrane. Over the time course of the trial no clinically significant $\omega-3$ PUFA indexes were reached which would be expected to exert any beneficial effects. An intravenous route with adaptable dosage should have been the preferred approach in the acute phase as used in the ongoing FOILED study (NCT01146821). In addition, the negative results could also be explained by the fact that the group treated with $\omega-3$ PUFA had a higher rate of multiple trauma patients [57 (91.9\%) vs. $45(77.6 \%)$, respectively, $P=0.04$ ], resulting in a higher rate of packed red blood cell transfusions (189 vs. 77 units, respectively, $P=0.03$ ) compared to controls.

Therefore, the results of this study should be interpreted with caution. The trial by Kagan and colleagues demonstrates once more that absence of evidence is not evidence of absence.

\section{Compliance with ethical standards}

Conflicts of interest Axel R. Heller received project funding and lecture fees from Fresenius Kabi Deutschland $\mathrm{GmbH}$ and BBraun Melsungen AG. Thomas Felbinger is a member of advisory boards and received additionally honoraria for lectures from the following companies: BBraun Melsungen AG, Baxter Deutschland $\mathrm{GmbH}$, Fresenius Kabi Deutschland $\mathrm{GmbH}$, and Nutritcia GmbH.

\section{References}

1. Kagan I, Cohen J, Stein M, Bendavid I, Pinsker D, Silva V, Theilla M, Anbar R, Lev S, Grinev M, Singer P (2015) Preemptive enteral nutrition enriched with eicosapentaenoic acid, gammalinolenic acid and antioxidants in severe multiple trauma: a prospective, randomized, double-blind study. Intensive Care Med 41:460-469. doi: 10.1007/s00134-015-3646-z

2. Pontes-Arruda A, Demichele S, Seth A, Singer P (2008) The use of an inflammation-modulating diet in patients with acute lung injury or acute respiratory distress syndrome: a metaanalysis of outcome data. J Parenter Enteral Nutr 32(6):596-605

3. Pontes-Arruda A, Aragao AM, Albuquerque JD (2006) Effects of enteral feeding with eicosapentaenoic acid, gamma-linolenic acid, and antioxidants in mechanically ventilated patients with severe sepsis and septic shock. Crit Care Med 34(9):2325-2333

4. Bower RH, Cerra FB, Bershadsky B, Licari JJ, Hoyt DB, Jensen GL et al (1995) Early enteral administration of a formula (Impact) supplemented with arginine, nucleotides, and fish oil in intensive care unit patients: results of a multicenter, prospective, randomized, clinical trial. Crit Care Med 23(3):436-449

\section{Uhlig $(\bullet)$ - A. R. Heller}

Department of Anesthesiology and Critical Care, University Hospital Dresden,

Technische Universität Dresden, Dresden, Germany e-mail: christopher.uhlig@ uniklinikum-dresden.de

\section{T. Felbinger}

Department of Anesthesiology, Critical

Care and Pain Therapy Klinikum

Neuperlach, Städtisches Klinikum München

GmbH, Munich, Germany 